\title{
The effect of splicing MST1R in gastric cancer was enhanced by IncRNA FENDRR
}

\author{
DONGHUI ZHOU ${ }^{1}$, XIAOHUA ZHU ${ }^{1}$, XUAN WU ${ }^{1}$, JINGJING ZHENG ${ }^{2}$, LAIZHEN TOU ${ }^{1}$ and YONG ZHOU ${ }^{1}$ \\ ${ }^{1}$ Department of Oncology, First Affiliated Hospital, Zhejiang University School of Medicine, Hangzhou, Zhejiang 310003; \\ ${ }^{2}$ Department of General Surgery, Lishui Municipal Central Hospital, Lishui, Zhejiang 323000, P.R. China
}

Received September 25, 2020; Accepted March 17, 2021

DOI: $10.3892 /$ etm.2021.10230

\begin{abstract}
Gastric cancer (GC) poses a serious threat to human health worldwide. Serine/arginine rich splicing factor 1 (SRSF1) has been reported to serve regulatory roles during the tumorigenesis of GC. In addition, the macrophage stimulating 1 receptor (MST1R) signaling pathway was found to participate in the progression of GC. However, the association between MST1R and SRSF1 in the tumorigenesis of GC remains unclear. The expression levels of MST1R and the recepteur d'origine nantais $(\mathrm{RON}) \Delta 160$ splicing variant were analyzed in cells using western blotting and immunofluorescence staining. Co-immunoprecipitation assays were used to investigate the interaction between SRSF1 and MST1R. A Cell Counting Kit- 8 assay was performed to analyze cell viability. Flow cytometry and Transwell assays were used to determine cell apoptosis and invasiveness levels. The potential interaction between SFSR1 and long non-coding RNAs (lncRNAs) was investigated with an online bioinformatics tool. The findings of the present study revealed that the expression levels of MST1R and RON $\Delta 160$ were significantly upregulated in GC Kato III cells. SRSF1 was found to be regulated by the lncRNA FOXF1 adjacent non-coding developmental regulatory RNA (FENDRR). The knockdown of SRSF1 or FENDRR downregulated the expression levels of MST1R in Kato III cells. In addition, the expression levels of RON $\Delta 160$ were markedly downregulated in Kato III cells following the knockdown of FENDRR. Meanwhile, SRSF1 directly bound to MST1R, while this phenomenon was partially reversed by FENDRR short interfering RNA. FENDRR could interact with SRSF1 in Kato III cells and the knockdown of FENDRR also induced the apoptosis of GC cells. In conclusion, the findings of the present study suggested that the lncRNA FENDRR may function as an oncogene during the progression of GC by
\end{abstract}

Correspondence to: Dr Donghui Zhou, Department of Oncology, First Affiliated Hospital, Zhejiang University School of Medicine, 79 Qingchun Road, Hangzhou, Zhejiang 310003, P.R. China E-mail: 1193076@zju.edu.cn

Key words: FOXF1-AS1, SRSF1, gastric cancer, MST1R regulating alternative splicing of MST1R and SRSF1 expression levels. lncRNA FENDRR may serve as a potential marker for the diagnosis or target for the treatment of GC.

\section{Introduction}

Gastric cancer (GC) is one of the leading causes of cancer-related mortality worldwide, demonstrating over $50 \%$ of morbidity and mortality (1). Despite significant advances, the overall survival rate of patients with GC has not improved in the past 20 years (2). Although numerous molecular targets have been reported to be involved in the progression of GC $(3,4)$, the mechanisms underlying GC development remain to be further investigated.

Serine/arginine rich splicing factor (SRSF) 1 is a $35-\mathrm{kDa}$ serine/arginine-rich splicing factor. SRSF1 can regulate pre-mRNA alternative splicing and maintain genome stability by interacting with transcription factor E2F transcription factor 1 in the cell cycle (5). Furthermore, SRSF1 facilitates transcriptional elongation by recruiting positive transcription elongation factor $\mathrm{b}$ kinase and promoting the subsequent phosphorylation of serine 2 on the C-terminal domain of RNA polymerase II at the post-transcriptional level (6).

Macrophage stimulating 1 receptor (MST1R) is a membrane tyrosine kinase of the MET family, which has been reported to be a novel potential target for cancer treatment (7). MST1R has been found to be positively associated with the development and progression of multiple types of epithelial cancer, including GC (8-10). Moreover, several preclinical studies have revealed that MST1R exhibited oncogenic properties, including the promotion of cellular proliferation, migration, invasion and survival in several human cancer cell lines $(11,12)$. Despite exhibiting diverse functions in numerous malignancies, the alternatively spliced products of MST1R show considerable sequence homology with each other (11). In human cancer, the alterative skipping of MST1R exon 5 and exon 6 promotes the loss of 106 amino acids in the MST1R $\beta$-chain, which produces a $109 \mathrm{kDa}$ MST1R splicing variant known as recepteur d'origine nantais (RON) $\Delta 160$. This isoform was identified to be involved in the activation of the PI3K/AKT signaling pathway and cellular transformation in vitro (13). Previous studies found that SRSF1 played a key role in the regulation of MST1R pre-mRNA splicing $(8,13)$. Therefore, further investigation into the association between 
SRSF1 and MST1R isoform splice variants in GC may provide novel insights for targeted therapies.

\section{Materials and methods}

Cell lines and culture. The GC cell lines Kato III and MKN-45 and the immortalized gastric epithelium cell line GES-1 were obtained from the State Key Laboratory for Diagnosis and Treatment of Infectious Diseases, The First Affiliated Hospital, Zhejiang University School of Medicine (Hangzhou, China). Cells were cultured in RPMI-1640 medium (Thermo Fisher Scientific, Inc.), supplemented with 10\% FBS (Thermo Fisher Scientific, Inc.), $100 \mu \mathrm{g} / \mathrm{ml}$ streptomycin and penicillin, and maintained at $37^{\circ} \mathrm{C}$ in $5 \% \mathrm{CO}_{2}$.

Vector construction and cell transfection. Small interfering RNA (siRNA) targeting SRSF1 (SRSF1-siRNA; $10 \mathrm{nM})$, FENDRR-siRNA (10 nM), pcDNA3.1 vector for MST1R overexpression (pcDNA3.1-MST1R, MST1R OE) and the corresponding empty vector [(referred to as negative control (NC)] were purchased from Sangon Biotech (Shanghai) Co., Ltd. SRSF1- and FENDRR-siRNA were transfected into GC cells using Lipofectamine ${ }^{\circledR} 2000$ reagent (Invitrogen; Thermo Fisher Scientific, Inc.) according to the manufacturer's protocol. The transfection efficiency was analyzed using reverse transcription-quantitative PCR (RT-qPCR). The sequences of the siRNAs were as follows: NC siRNA (siRNA-ctrl), 5'-UUCUCCGAACGUGUCACGUTT-3'; SRSF1-siRNA, 5'-GGAAUGAAGCAACUGAGAUUU-3'; and FENDRR-siRNA, 5'-GGGTTACGATTGCCCAGAT-3'. For MST1R overexpression, GC cells were plated into $60-\mathrm{mm}$ plates at a density of $4 \times 10^{5}$ cells/well and incubated overnight. Upon cells reaching 50-60\% confluence, supernatants containing the pcDNA3.1 vector carrying the MST1R gene were added directly to the cell culture and incubated for $24 \mathrm{~h}$. Following the incubation, GC cells were plated in selection medium containing $2.5 \mu \mathrm{g} / \mathrm{ml}$ puromycin for a further 3 days.

Cell Counting Kit-8 (CKK-8) assay. A CCK-8 assay (Beyotime Institute of Biotechnology) was used to determine cell viability. Briefly, GC cells were plated into 96-well plates at a density of $5 \times 10^{3}$ cells/well and transfected with NC, siRNA-FENDRR or siRNA-FENDRR + MST1R pcDNA3.1 vector for $72 \mathrm{~h}$. Following the incubation, cells were incubated with $10 \mu \mathrm{l}$ CCK-8 reagent for another $2 \mathrm{~h}$ at $37^{\circ} \mathrm{C}$. The absorbance of each well was measured at a wavelength of $450 \mathrm{~nm}$ using a microplate reader (Thermo Fisher Scientific, Inc.).

Cell apoptosis. GC cells were seeded into six-well plates $\left(5 \times 10^{4}\right.$ cells/well). Following centrifugation at $200 \mathrm{x}$ g for $5 \mathrm{~min}$ at $4^{\circ} \mathrm{C}$, the cell pellet was resuspended in $100 \mu \mathrm{l}$ binding buffer and incubated with $5 \mu \mathrm{l}$ Annexin V-FITC (BD Biosciences) and propidium iodide (BD Biosciences) at room temperature for $15 \mathrm{~min}$. The cell apoptotic rate was measured using a flow cytometer (BD Biosciences) and the data were analyzed using WinMDI 2.9 software.

Agarose electrophoresis. Vectors at a concentration of $0.03 \mu \mathrm{g}$ $\mathrm{DNA} / \mu 1$ were subjected to electrophoresis on an ethidium bromide-containing gel (1\% agarose). Subsequently, bands were photographed with a Vilber E-BOX (Vilber Lourmat Sté). Meanwhile, 1 unit DNase and $1.2 \mu \mathrm{g}$ DNA (Sigma-Aldrich; Merck $\mathrm{KGaA}$ ) were incubated at $37^{\circ} \mathrm{C}$ with the vectors and complexes for $30 \mathrm{~min}$. Subsequently, 2\% SDS solution was added as a DNA release reagent. Samples were subjected to agarose gel electrophoresis and compared with untreated DNA. The data were quantified using Image Pro Plus (version 6.0; Media Cybernetics, Inc.).

$R T-q P C R$. Total RNA was extracted from cell lines using TRIzol ${ }^{\circledR}$ reagent (Invitrogen; Thermo Fisher Scientific, Inc.). Total RNA was reverse transcribed into cDNA using a PrimeScript RT Reagent kit (Takara Bio, Inc.) according to the manufacturer's protocol. qPCR was subsequently analyzed using a SYBR Premix Ex Taq II kit (Takara Bio, Inc.) on a 7900HT system (Applied Biosystems; Thermo Fisher Scientific, Inc.) according to the following conditions: $60^{\circ} \mathrm{C}$ for $1 \mathrm{~min}, 90^{\circ} \mathrm{C}$ for $15 \mathrm{~min}$, followed by 40 cycles of application at $90^{\circ} \mathrm{C}$ for $15 \mathrm{sec}$ and $55^{\circ} \mathrm{C}$ for $60 \mathrm{sec}$. The following primer sequences were used for the qPCR: FENDRR forward, 5'-TTCATCGGCTGCGTATTCG-3' and reverse, 5'-TTGCCTTCTAGTCGCCTCC-3'; and $\beta$-actin forward, 5'-GTCCACCGCAAATGCTTCTA-3' and reverse, 5'-TGCTGTCACCTTCACCGTTC-3'. Expression levels were quantified using the $2^{-\Delta \Delta C q}$ method (14). $\beta$-actin was used as the internal control for normalization.

Transwell assay. A Transwell assay was performed to analyze cell migration and invasion. The upper chambers of the Transwell plates were pretreated with $100 \mu 1$ Matrigel (BD Biosciences) for $4 \mathrm{~h}$ at $37^{\circ} \mathrm{C}$, while Matrigel was not used in the migration assay GC cells were seeded into the upper chamber of the plates in media supplemented with $1 \%$ FBS at a density of $1 \times 10^{6}$ cells/chamber. RPMI-1640 medium supplemented with $10 \%$ FBS was added into the lower chambers. Following $24 \mathrm{~h}$ of incubation at $37^{\circ} \mathrm{C}$, the Transwell chamber was rinsed twice with PBS (5 min each time), then the cells were fixed with $5 \%$ glutaraldehyde at $4^{\circ} \mathrm{C}$ and stained at $37^{\circ} \mathrm{C}$ with $0.1 \%$ crystal violet for $30 \mathrm{~min}$. The Transwell chamber was washed twice with PBS and observed under a light microscope (magnification, $\mathrm{x} 200$ ). Three random fields were selected. The number of cells invading the Matrigel was a reflection of the invasive ability.

Western blotting. Total protein was extracted from cells using RIPA lysis buffer (Beyotime Institute of Biotechnnology) and quantified using a BCA protein assay kit (Beyotime Institute of Biotechnology). Proteins (40 $\mu \mathrm{g}$ per lane) were separated via $10 \%$ SDS-PAGE, then transferred onto PVDF membranes (Bio-Rad Laboratories, Inc.). After blocking with $5 \%$ skimmed milk for $1 \mathrm{~h}$ at room temperature, the membranes were incubated with the following primary antibodies at $4^{\circ} \mathrm{C}$ overnight: Anti-AKT (cat. no. ab18785; 1:1,000), anti-phosphorylated (p)-AKT (cat. no. ab38449; 1:1,000), Anti-p-ERK (cat. no. ab201015; 1:1,000), anti-ERK (cat. no. ab32081; 1:1,000), anti-MST1R (cat. no. ab52927; 1:1,000), anti-cleaved caspase-3 (cat. no. ab32042; 1:1,000), anti-SRSF1 (Thermo Fisher Scientific, Inc.; cat. no. 32-4500, 1:1,000) and anti- $\beta$-actin (cat. no. ab8226; 1:1,000; all Abcam except for SRSF1). Following the primary antibody incubation, the membranes were incubated with an anti-rabbit secondary 
antibody (HRP-conjugated; Abcam; cat. no. ab7090, 1:5,000) at room temperature for $1 \mathrm{~h}$. Enhanced chemiluminescence reagent (Thermo Fisher Scientific, Inc.) was used to visualize the protein bands. ImageJ software (version 2.0; National Institutes of Health) was used to quantify the intensity of the bands.

Bioinformatics prediction. The LncRNA2Targetv2.0 tool (http://123.59.132.21/1ncrna2target/index.jsp) was used to determine the interaction between long non-coding RNAs (lncRNAs) and upstream of the SRSF1 coding region.

RNA pull-down. For the RNA pulldown assay, Biotin RNA Labeling Mix (Roche Diagnostics) was used to transcribe and label probe-control or probe-FENDRR in vitro. An RNA structure buffer (Thermo Fisher Scientific, Inc.) was used to induce secondary structure formation from the biotin-labeled RNAs. The biotinylated FENDRR (Shanghai GenePharma Co., Ltd.) and negative control (bio-NC; GenePharma) were coated with streptavidin-conjugated magnetic beads (Roche Diagnostics). GC cells were lysed using lysis reagent (Roche Diagnostics) and then incubated with the magnetic beads for $6 \mathrm{~h}$. The enrichment level of SRSF1 was detected by western blotting.

Immunofluorescence staining. GC cells were fixed with $4 \%$ paraformaldehyde for $10 \mathrm{~min}$ at room temperature and then fixed with pre-cooled methanol at $4^{\circ} \mathrm{C}$ for a further $10 \mathrm{~min}$. Subsequently, cells were incubated with an anti-RON $\Delta 160$ primary antibody (Abcam; cat. no. ab124671, 1:1,000) overnight at $4{ }^{\circ} \mathrm{C}$. Following the primary antibody incubation, the cells were incubated with a goat anti-rabbit IgG secondary antibody (Abcam; cat. no. ab6721, 1:5,000) for $1 \mathrm{~h}$ at room temperature. DAPI (Beyotime Institute of Biotechnology) was used to counterstain the nuclei. Samples were visualized using a fluorescence microscope (model no. CX23; Olympus Corporation, magnification, $\mathrm{x} 200$ ). Three random fields were selected.

Co-immunoprecipitation (co-IP) assay. The co-IP assay was performed as previously described with modifications (5). Briefly, Kato III cell protein supernatants were pretreated with $50 \mu \mathrm{l} \mathrm{A} / \mathrm{G}$ (Protein A/Protein G) beads (Selleck Chemicals) prior to immunoprecipitation and then with $5 \mu \mathrm{g}$ control $\mathrm{IgG}$ (Santa Cruz Biotechnology, Inc.), anti-MST1R or anti-SRSF1 magnetic beads (Shanghai ZE YE Biological Technology Co., Ltd.) overnight at $4^{\circ} \mathrm{C}$. Following further incubation with $50 \mu \mathrm{l}$ $\mathrm{A} / \mathrm{G}$ beads at $4^{\circ} \mathrm{C}$ for $6 \mathrm{~h}$, the immunoprecipitates were eluted with ice-cold PBS supplemented with $0.2 \%$ NP-40 five times. Subsequently, the immunoprecipitated proteins were separated via SDS-PAGE and visualized using western blotting as aforementioned.

Statistical analysis. Data are presented as the mean \pm SD. Statistical differences between two groups were determined using unpaired Student's t-test. Comparisons among multiple groups were made using an ANOVA followed by a Tukey's post hoc test. Statistical analysis was performed using GraphPad Prism 7 software (GraphPad Software, Inc.). P $<0.05$ was considered to indicate a statistically significant difference.

\section{Results}

Expression levels of MSTIR and RON $\triangle 160$ are upregulated in Kato III cells. To determine the biological function of MST1R and RON $\Delta 160$ in GC, western blotting was performed. As indicated in Fig. 1A, the expression levels of MST1R were significantly upregulated in Kato III cells compared with the other cell lines. The expression levels of RON $\Delta 160$ in Kato III cells were higher compared with GES-1 cells (Fig. 1B). Moreover, the mRNA expression levels of MST1R and RON $\Delta 160$ were also notably upregulated in Kato III cells compared with GES-1 cells (Fig. 1C). Therefore, Kato III cells were selected for use in subsequent experiments. These results revealed that the expression levels of MST1R and RON $\Delta 160$ may be upregulated in Kato III cells.

Knockdown of SRSF1 significantly downregulates MST1R and RON $\Delta 160$ expression levels in Kato III cells. To determine the transfection efficiency of SRSF1-siRNA, RT-qPCR was performed. As shown in Fig. 2A and B, SRSF1 expression levels were significantly downregulated in Kato III cells following SRSF1 knockdown. Moreover, Kato III cells were more sensitive to SRSF1-2-siRNA. Thus, SRSF1-2- siRNA was selected for use in subsequent experiments. Similarly, the expression levels of RON $\Delta 160$ were significantly downregulated following the transfection with SRSF1-2- siRNA in Kato III cells (Fig. 2C). The expression levels of MST1R were also downregulated in GC cells following the knockdown of SRSF1 (Fig. 2D). As expected, the mRNA expression levels of MST1R and RON $\triangle 160$ in GC cells were also notably downregulated following transfection with SRSF1-2-siRNA (Fig. 2E). Taken together, these findings indicated that the knockdown of SRSF1 may significantly downregulate MST1R and RON $\Delta 160$ in Kato III cells.

Knockdown of FENDRR suppresses the expression levels of MSTIR and alters the distribution of RON $\triangle 160$ in Kato III cells. FENDRR was identified to directly interact with SRSF1. RT-qPCR was used to analyze the transfection efficiency of FENDRR-siRNA transfection. As shown in Fig. 3A, the expression levels of FENDRR were downregulated in Kato III cells in the presence of FENDRR-siRNA. In addition, the knockdown of FENDRR significantly downregulated the expression levels of SRSF1 and MST1R in Kato III cells (Fig. 3B). Immunofluorescence staining revealed that RON $\Delta 160$ expression was significantly upregulated in Kato III cells in the presence of FENDRR-siRNA (Fig. 3C). mRNA expression levels of MST1R and RON $\Delta 160$ in Kato III cells were also markedly upregulated following the transfection with FENDRR-siRNA (Fig. 3D). Altogether, these findings suggested that the silencing of FENDRR may regulate the expression levels of MST1R and alter the distribution of RON $\Delta 160$ in Kato III cells by suppressing SRSF1 expression.

Silencing of FENDRR suppresses the proliferation of GC cells by regulating MSTIR. To determine the effect of FENDRR siRNA on interaction between SRSF1 and MST1R, co-IP assays were performed. As demonstrated in Fig. 4A, SRSF1 was found to directly bind with MST1R, while FENDRR siRNA reversed this phenomenon. Meanwhile, FENDRR 

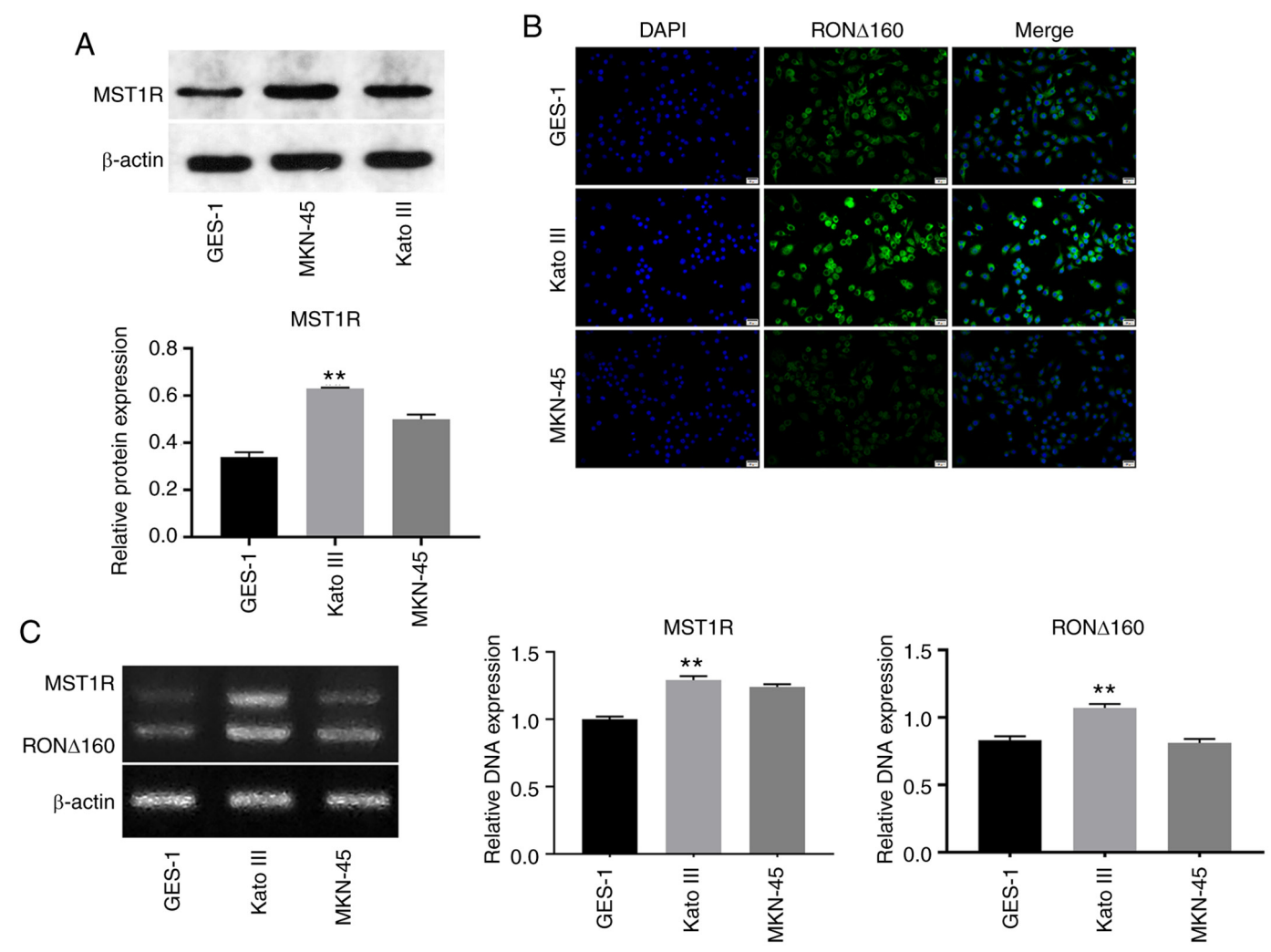

Figure 1. MST1R and RON $\Delta 160$ are upregulated in Kato III cells. (A) The protein expression levels of MST1R in GES-1, Kato III and MKN-45 cells were determined by western blot. The relative expression was quantified by normalizing to $\beta$-actin. (B) The expression levels of RON $\Delta 160$ in GES-1, Kato III and MKN-45 cells were investigated by immunofluorescence staining. Green fluorescence indicates RON $\Delta 160$. Blue fluorescence indicates DAPI. (C) Electrophoresis of MST1R, RON $\Delta 160$ and $\beta$-actin polymerase chain reaction products on the agarose gel showed a unique band with expected sizes for each gene for GES-1, Kato III and MKN-45 cells. DNA expression was quantified by normalizing to $\beta$-actin. MST1R, macrophage stimulating 1 receptor; RON $\triangle 160$, recepteur d'origine nantais. ${ }^{* *} \mathrm{P}<0.01$ vs. GES-1 cells.
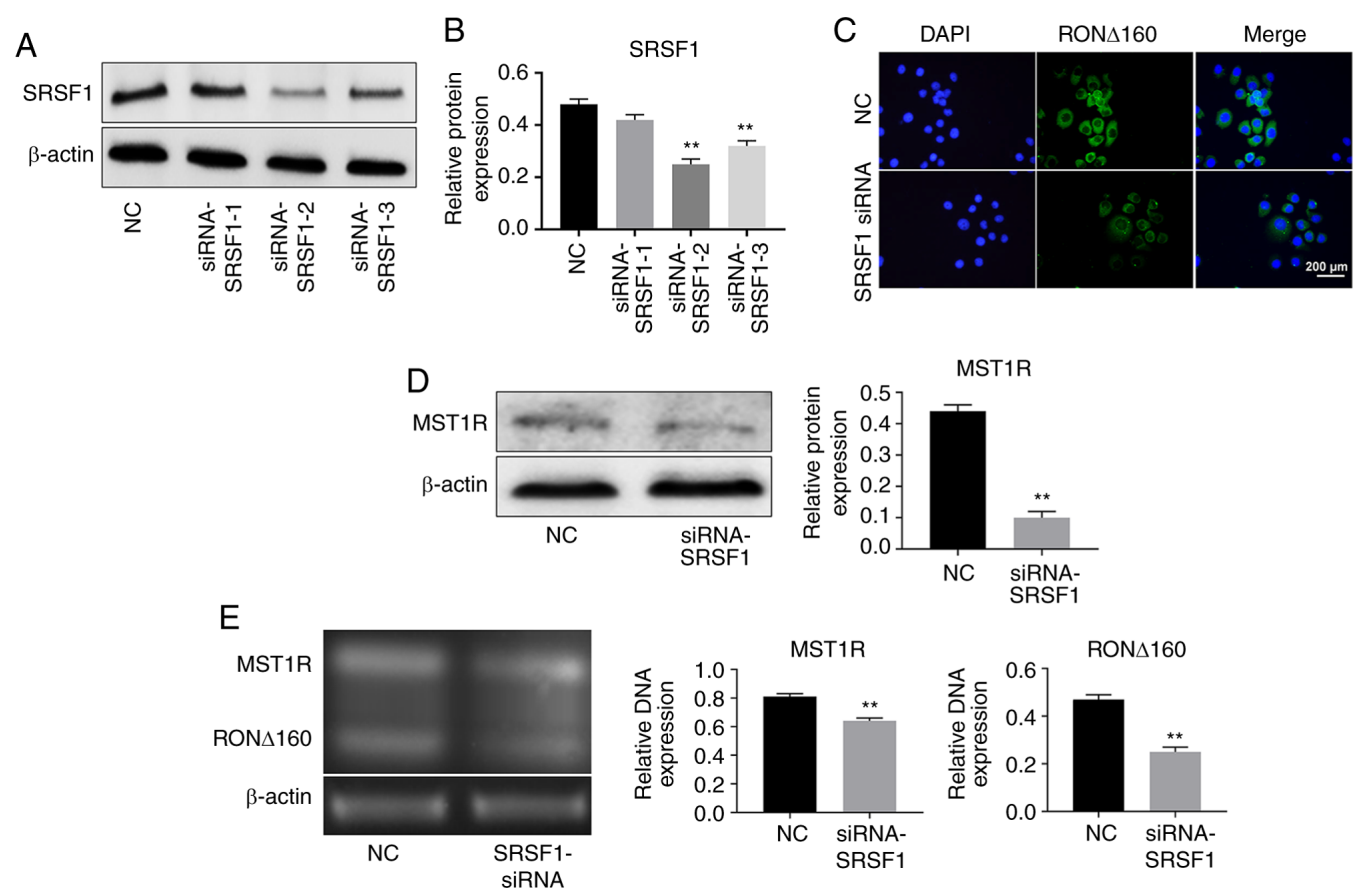

Figure 2. Knockdown of SRSF1 significantly inactivates MST1R and RON $\Delta 160$ in Kato III cells. (A) Kato III cells were transfected with NC, siRNA-SRSF1-1, siRNA-SRSF1-2 or siRNA-SRSF1-3 for $24 \mathrm{~h}$ and then western blotting was used to detect the efficiency of transfection. (B) The relative protein expression of SRSF1 was quantified by normalizing to $\beta$-actin. (C) The expression of RON $\Delta 160$ in Kato III cells was measured by immunofluorescence staining. (D) The protein expression level of MST1R in Kato III cells was determined by western blot. The relative expression levels of MST1R were quantified by normalizing to $\beta$-actin. (E) Electrophoresis of MST1R, RON $\Delta 160$ and $\beta$-actin polymerase chain reaction products on the agarose gel showed a unique band with expected sizes for each gene for Kato III cells. DNA expression was quantified by normalizing to $\beta$-actin. SRSF1, serine/arginine rich splicing factor 1; MST1R, macrophage stimulating 1 receptor; RON $\Delta 160$, recepteur d'origine nantais; $\mathrm{NC}$, non-coding control; siRNA, short interfering RNA. ${ }^{* *} \mathrm{P}<0.01$ vs. control. 


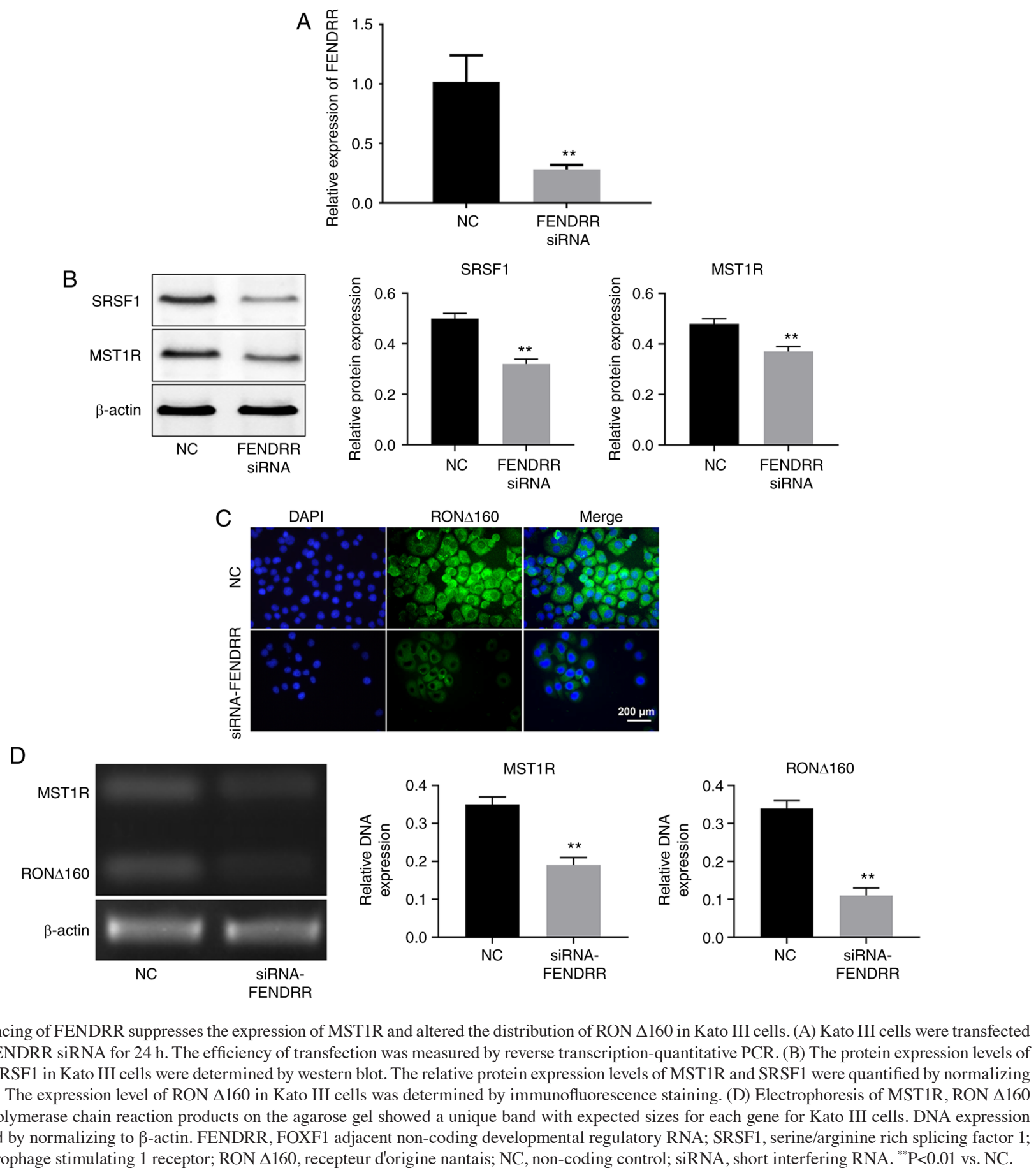

Figure 3. Silencing of FENDRR suppresses the expression of MST1R and altered the distribution of RON $\Delta 160$ in Kato III cells. (A) Kato III cells were transfected with NC or FENDRR siRNA for $24 \mathrm{~h}$. The efficiency of transfection was measured by reverse transcription-quantitative PCR. (B) The protein expression levels of MST1R and SRSF1 in Kato III cells were determined by western blot. The relative protein expression levels of MST1R and SRSF1 were quantified by normalizing to $\beta$-actin. (C) The expression level of RON $\Delta 160$ in Kato III cells was determined by immunofluorescence staining. (D) Electrophoresis of MST1R, RON $\Delta 160$ and $\beta$-actin polymerase chain reaction products on the agarose gel showed a unique band with expected sizes for each gene for Kato III cells. DNA expression was quantified by normalizing to $\beta$-actin. FENDRR, FOXF1 adjacent non-coding developmental regulatory RNA; SRSF1, serine/arginine rich splicing factor 1 ; MST1R, macrophage stimulating 1 receptor; RON $\Delta 160$, recepteur d'origine nantais; NC, non-coding control; siRNA, short interfering RNA. ${ }^{* *} \mathrm{P}<0.01$ vs. NC.

could bind with SRSF1 (Fig. 4B). A CCK-8 assay was used to analyze cell viability. The results revealed that the knockdown of FENDRR notably inhibited the viability of Kato III cells, while the reduction in viability induced by FENDRR-siRNA was partially rescued by the overexpression of MST1R (Fig. 5A). Similarly, the apoptosis of Kato III cells was markedly increased following the genetic silencing of FENDRR, which was significantly reversed by the overexpression of MST1R (Fig. 5B). In addition, Kato III cell migration and invasion levels were suppressed in the presence of FENDRR-siRNA. However, the inhibitory effect of FENDRR-siRNA on cell invasion was significantly abrogated by MST1R overexpression (Fig. 5C and D). Altogether, these findings suggested that the knockdown of FENDRR may suppress the proliferation of GC cells by regulating MST1R expression.

Knockdown of FENDRR significantly inhibits the progression of $G C$ in vitro via inactivation of PI3K/AKT signaling. To further investigate the mechanism through which FENDRR mediated the progression of GC, western blotting was performed. As shown in Fig. 6A-E, the protein expression levels of MST1R and the p-AKT/AKT and p-ERK/ERK ratios were notably downregulated in Kato III cells following the knockdown of FENDRR, and were partially reversed following MST1R overexpression. In contrast, the genetic silencing of FENDRR markedly upregulated the ratio of cleaved caspase-3/procaspase-3 in GC cells, while the promoting effect 


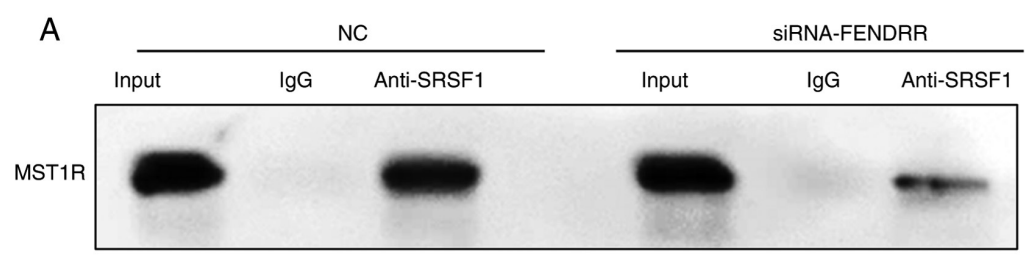

B
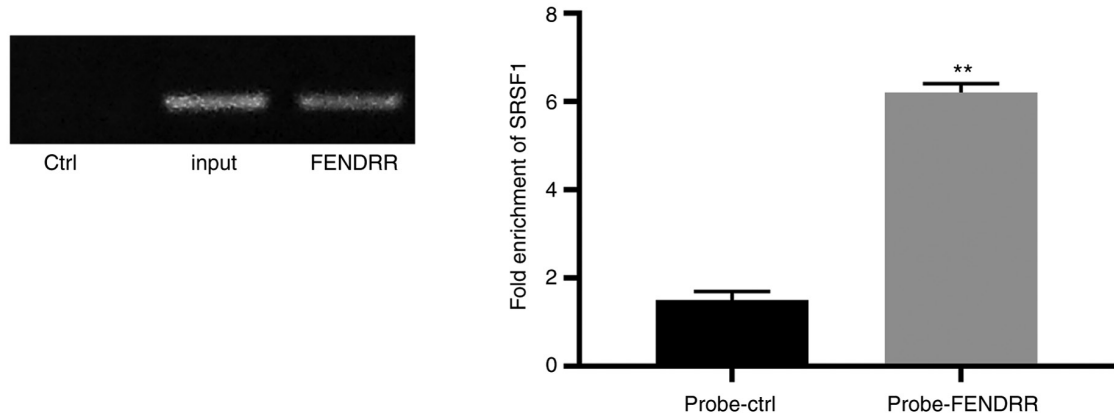

Figure 4. SRSF1 binds to MST1R. (A) Equal amounts of total lysates were applied to co-immunoprecipitation with IgG, anti-SRSF1 or anti-MST1R magnetic beads. Immunoprecipitation analysis of Kato III cells transfected with NC or siRNA- FENDRR . (B) The enrichment of SRSF1 was detected by RNA pull-down. FENDRR, FOXF1 adjacent non-coding developmental regulatory RNA; SRSF1, serine/arginine rich splicing factor 1; MST1R, macrophage stimulating 1 receptor; NC, non-coding control; siRNA, short interfering RNA; IgG, immunoglobulin G; ctrl, control. ${ }^{* * *} \mathrm{P}<0.01$ vs. probe-ctrl.

A $\simeq$

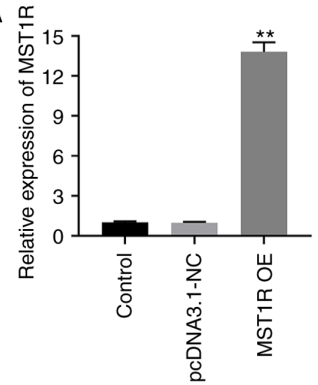

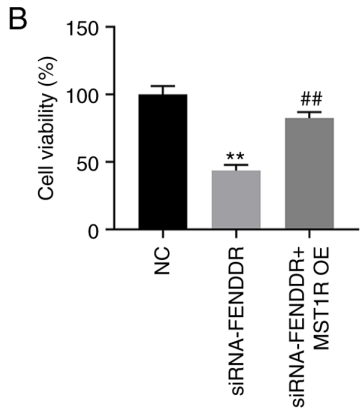

C

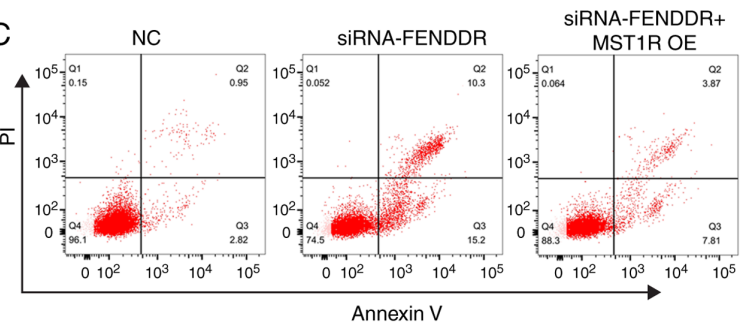

$\mathrm{D}$

NC

SiRNA-FENDDR
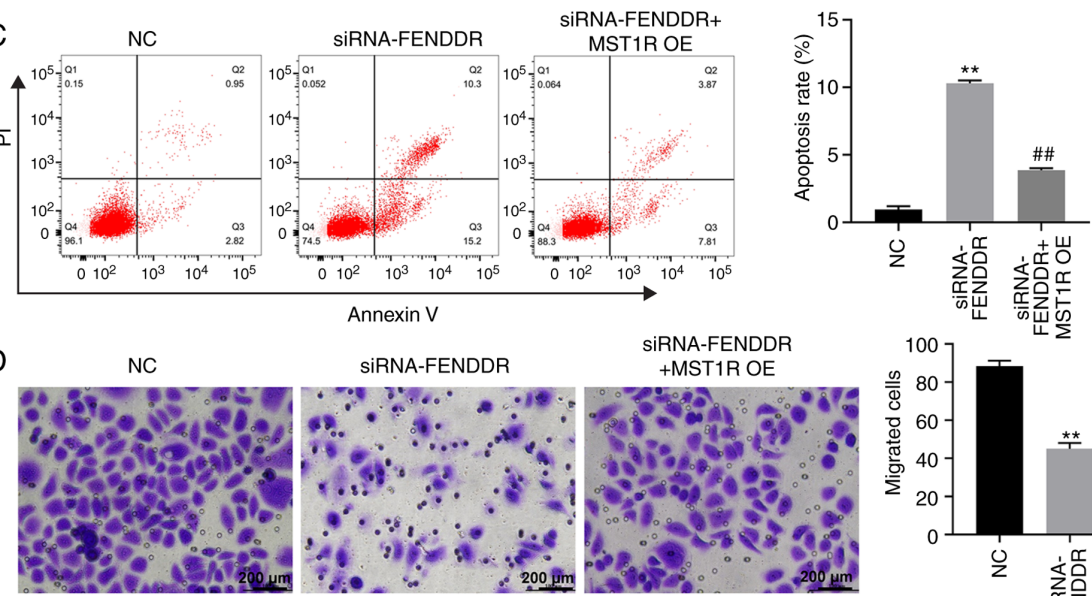

E
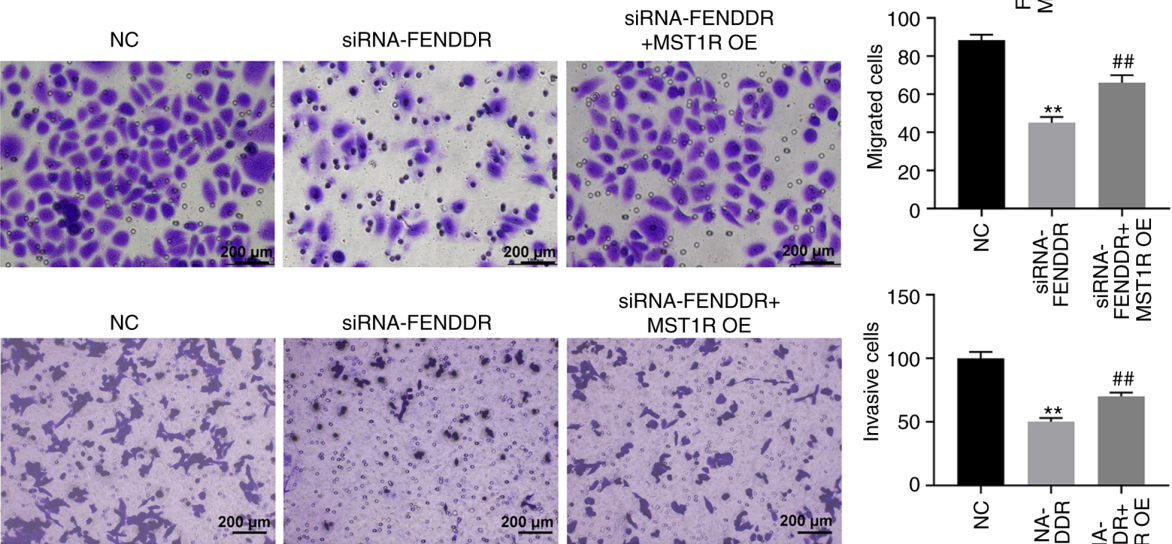

SiRNA-FENDDR+
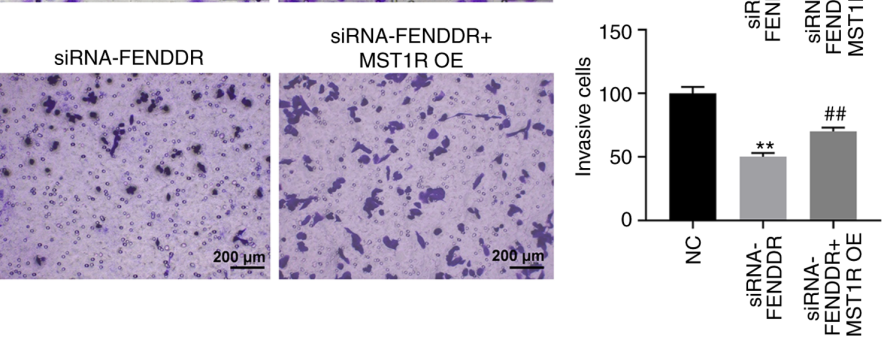

Figure 5. Silencing of FENDRR suppresses the growth of gastric cells via MST1R. (A) Kato III cells were transfected with pcDNA3.1 (NC) or MST1R OE. The expression of MST1R in Kato III cells was determined by reverse transcription-quantitative PCR. (B) Cells were treated with NC, siRNA-FENDRR or FENDRR + MST1R OE for $72 \mathrm{~h}$. Cell viability was then tested. (C) The apoptosis rate in Kato III cells was measured by flow cytometry after double staining with Annexin V and PI. (D) Cell migration and (E) invasion were assessed by transwell assay. FENDRR, FOXF1 adjacent non-coding developmental regulatory RNA; MST1R, macrophage stimulating 1 receptor; OE, overexpression vector; NC, non-coding control; siRNA, short interfering RNA; PI, propidium iodide. ${ }^{* *} \mathrm{P}<0.01$ vs. control, ${ }^{\#} \mathrm{P}<0.01$ vs. siRNA-FENDRR. 

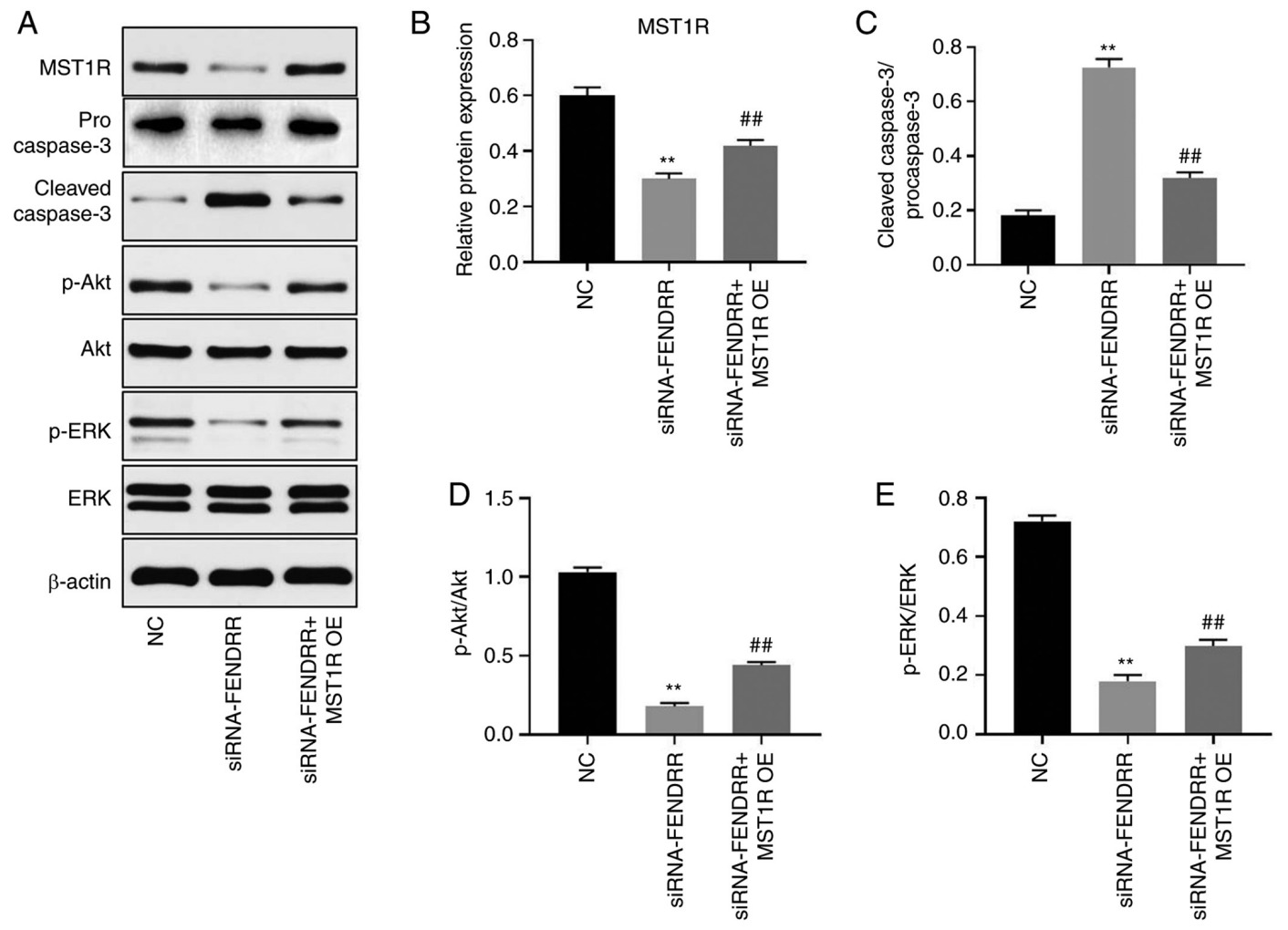

Figure 6. Silencing of FENDRR inhibits the tumorigenesis of gastric cancer cells in vitro via inactivation of PI3K/Akt signaling. (A) The protein expression levels of MST1R, caspase-3, p-Akt, Akt, p-ERK and ERK in Kato III cells were determined by western blot. (B) The relative protein expression of MST1R was quantified by normalizing to $\beta$-actin. (C) The ratio of cleaved caspase-3/procaspase-3 was calculated. (D) The ratio of p-Akt/Akt was calculated. (E) The ratio of p-ERK/ERK was calculated. FENDRR, FOXF1 adjacent non-coding developmental regulatory RNA; MST1R, macrophage stimulating 1 receptor; OE, overexpression vector; NC, non-coding control; siRNA, short interfering RNA; ERK, extracellular signal related kinase; p, phosphorylated ${ }^{* *} \mathrm{P}<0.01$ vs. NC; ${ }^{\# \#} \mathrm{P}<0.01$ vs. siRNA-FENDRR.

of FENDRR-siRNA on the cleaved caspase-3/procaspase-3 ratio was notably abrogated following MST1R overexpression. Altogether, these results suggested that the knockdown of FENDRR may inhibit the progression of GC in vitro by inhibiting PI3K/AKT signaling.

\section{Discussion}

MST1R has been reported to play a key role in the pathogenesis of GC (9). The alternative splicing variant of precursor MST1R mRNA, RON $\Delta 160$, has been identified as an oncogenic transcription factor that regulates numerous signaling pathways associated with tumorigenesis, including cellular transformation activities such as focus formation and anchorage-independent growth (8). A previous study demonstrated that the upregulation of variant RON $\Delta 160$ in GC altered the phenotype and enhanced the invasive ability of GC cells. Furthermore, RON $\Delta 160$ was found to be closely associated with tumorigenesis, and both regional lymph node and widespread metastasis (13). The findings of the present study revealed that the expression levels of MST1R were regulated by FENDRR knockdown. These findings further validated the results of previous studies $(8,13)$, indicating that FENDRR and SRSF1 may promote the tumorigenesis of $\mathrm{GC}$ by regulating RON $\Delta 160$ expression.

SRSF1 is known to serve roles in RNA splicing and genome stability $(6,15,16)$. The RNA recognition motif of SRSF1 for RNA binding can promote spliceosome assembly at adjacent splice sites to facilitate appropriate exon inclusion $(17,18)$. The aberrant spliceosome function of SRSF1 was previously associated with the mis-splicing of multiple genes, including MST1R and enhancer of zeste 2 polycomb repressive complex 2 subunit, which have been implicated in the pathogenesis of myeloid neoplasms $(19,20)$. The results of the current study demonstrated that the knockdown of SRSF1 could lead to the inactivation of MST1R and RON $\triangle 160$ in GC cells. In addition, the results of the co-IP assay found that SRSF1 directly bound to MST1R. These data provided novel insights into the biological function of SRSF1, suggesting that SRSF1 may regulate MST1R and RON $\Delta 160$ expression. According to Bonomi et al (21), the involvement of SRSF1 in epithelial-to-mesenchymal transition derives from its ability to affect the splicing program of the proto-oncogene MST1R. Previous research has shown that SRSF1 could promote the production of MST1R through skipping of exon 11. More specifically, SRSF1 acts by directly binding to an exonic splicing enhancer (ESE) located in the constitutive exon 12 (22). Therefore, SRSF1 may interact with MST1R in GC.

An increasing number of studies have reported the important role of non-coding RNAs in cellular biological functions $(23,24)$. Previous research indicated that FENDRR was associated with the development of osteosarcoma (25). The present study found that the knockdown of FENDRR downregulated MST1R and RON $\Delta 160$ expression levels in GC cells. Based on these findings, it was hypothesized that the knockdown of FENDRR expression may result in the downregulation of RON $\Delta 160$ in GC. 
Furthermore, the current research revealed that the genetic silencing of FENDRR inactivated the PI3K/AKT signaling pathway in GC cells. PI3K/AKT signaling is known to be involved in the tumorigenesis of cancer $(26,27)$. A previous study reported that PI3K/AKT signaling played a key role in cancer progression, drug resistance and treatment (28). $\mathrm{Xu}$ et al (29) found that PI3K/AKT signaling led to reduced apoptosis and increased proliferation in GC cells. The findings of the present study were consistent with the aforementioned studies. Moreover, the present study demonstrated that MST1R overexpression partially rescued the inhibitory effect of FENDRR-siRNA on the PI3K/AKT signaling pathway in vitro. Ling et al (30) demonstrated that MST1R promoted PI3K/AKT signaling during the development of colorectal cancer. These findings were consistent with the present data, suggesting that FENDRR may mediate the expression levels of MST1R and RON $\Delta 160$ by inhibiting PI3K/AKT signaling.

In conclusion, the results of the present study suggested that lncRNA FENDRR may function as an oncogene during the progression of GC by mediating the alternative splicing of MST1R and SRSF1 expression. Therefore, IncRNA FENDRR may serve as a potential target for the diagnosis and treatment of GC.

\section{Acknowledgements}

Not applicable.

\section{Funding}

This study was supported by a grant from the National Natural Science Foundation of China (grant no. 81272680) and Zhejiang Provincial Department of Science and Technology (grant no. 2020C03112).

\section{Availability of data and materials}

The datasets used and/or analyzed during the current study are available from the corresponding author on reasonable request.

\section{Authors' contributions}

DZ and XW conceived and supervised the study. DZ, XZ and XW designed the study. LT, JZ and YZ performed the experiments and analyzed the data. DZ, XZ and XW were responsible for confirming the authenticity of all the raw data. All authors reviewed the results and approved the final version of the manuscript.

\section{Ethics approval and consent to participate}

Not applicable

\section{Patient consent for publication}

Not applicable.

\section{Competing interests}

These authors declare that they have no competing interests.

\section{References}

1. Rawicz-Pruszyński K, van Sandick JW, Mielko J, Ciseł B and Polkowski WP: Current challenges in gastric cancer surgery: European perspective. Surg Oncol 27: 650-656, 2018.

2. Miao L, Qi J, Zhao Q, Wu QN, Wei DL, Wei XL, Liu J, Chen J, Zeng ZL, Ju HQ, et al: Targeting the STING pathway in tumor-associated macrophages regulates innate immune sensing of gastric cancer cells. Theranostics 10: 498-515, 2020.

3. Barok M, Le Joncour V, Martins A, Isola J, Salmikangas M, Laakkonen P and Joensuu H: ARX788, a novel anti-HER2 antibody-drug conjugate, shows anti-tumor effects in preclinical models of trastuzumab emtansine-resistant HER2-positive breast cancer and gastric cancer. Cancer Lett 473: 156-163, 2020.

4. Wang Y, Lu JH, Wang F, Wang YN, He MM, Wu QN, Lu YX, Yu HE, Chen ZH, Zhao Q, et al: Inhibition of fatty acid catabolism augments the efficacy of oxaliplatin-based chemotherapy in gastrointestinal cancers. Cancer Lett 473: 74-89, 2020.

5. Anczuków O, Akerman M, Cléry A, Wu J, Shen C, Shirole NH, Raimer A, Sun S, Jensen MA, Hua Y, et al: SRSF1-regulated alternative splicing in breast cancer. Mol Cell 60: 105-117, 2015.

6. Comiskey DF Jr, Montes M, Khurshid S, Singh RK and Chandler DS: SRSF2 regulation of MDM2 reveals splicing as a therapeutic vulnerability of the p53 pathway. Mol Cancer Res 18: 194-203, 2020.

7. Yoon JY, Wang JY and Roehrl MH: A combined FAK, c-MET, and MST1R three-protein panel risk-stratifies colorectal cancer patients. Transl Oncol 13: 100836, 2020.

8. Krishnaswamy S, Mohammed AK, Tripathi G, Alokail MS and Al-Daghri NM: Splice variants of the extracellular region of RON receptor tyrosine kinase in lung cancer cell lines identified by PCR and sequencing. BMC Cancer 17: 738, 2017.

9. Chakedis J, French R, Babicky M, Jaquish D, Howard H, Mose E, Lam R, Holman P, Miyamoto J, Walterscheid Z, et al: A novel protein isoform of the RON tyrosine kinase receptor transforms human pancreatic duct epithelial cells. Oncogene 35: 3249-3259, 2016.

10. Milan M, Benvenuti S, Balderacchi AM, Virzì AR, Gentile A, Senetta R, Cassoni P, Comoglio PM and Stella GM: RON tyrosine kinase mutations in brain metastases from lung cancer. ERJ Open Res 4: 4, 2018.

11. Zhao S, Cao L and Freeman JW: Knockdown of RON receptor kinase delays but does not prevent tumor progression while enhancing HGF/MET signaling in pancreatic cancer cell lines. Oncogenesis 2: e76, 2013.

12. Paronetto MP, Passacantilli I and Sette C: Alternative splicing and cell survival: From tissue homeostasis to disease. Cell Death Differ 23: 1919-1929, 2016.

13. Zhou DH, Li C and Yang LN: Variant RON $\Delta 160$ of the RON receptor tyrosine kinase promotes the growth and invasion in vitro and in vivo in gastric cancer cell lines. Cancer Cell Int 15: 9, 2015.

14. Livak KJ and Schmittgen TD: Analysis of relative gene expression data using real-time quantitative PCR and the 2(- $\Delta \Delta \mathrm{C}(\mathrm{T}))$ method. Methods 25: 402-408, 2001.

15. Yoon M and Rikkerink EHA: Rpa1 mediates an immune response to avrRpm1Psa and confers resistance against Pseudomonas syringae pv. actinidiae. Plant J 102: 688-702, 2019.

16. Zhang D, Liu K, Hu W, Lu X, Li L, Zhang Q, Huang H and Wang H: Prenatal dexamethasone exposure caused fetal rats liver dysplasia by inhibiting autophagy-mediated cell proliferation. Toxicology 449: 152664, 2021.

17. van Bergeijk P, Seneviratne U, Aparicio-Prat E, Stanton R and Hasson SA: SRSF1 and PTBP1 are trans-acting factors that suppress the formation of a CD33 splicing isoform linked to Alzheimer's disease Risk. Mol Cell Biol 39: 39, 2019.

18. Coomer AO, Black F, Greystoke A, Munkley J and Elliott DJ: Alternative splicing in lung cancer. Biochim Biophys Acta Gene Regul Mech 1862: 1943882019.

19. Barbagallo D, Caponnetto A, Brex D, Mirabella F, Barbagallo C, Lauretta G, Morrone A, Certo F, Broggi G, Caltabiano R, et al: CircSMARCA5 regulates VEGFA mRNA splicing and angiogenesis in glioblastoma multiforme through the binding of SRSF1. Cancers (Basel) 11: 11, 2019.

20. Zhou X, Wang R, Li X, Yu L, Hua D, Sun C, Shi C, Luo W, Rao C, Jiang Z, et al: Splicing factor SRSF1 promotes gliomagenesis via oncogenic splice-switching of MYO1B. J Clin Invest 129: 676-693, 2019.

21. Bonomi S, di Matteo A, Buratti E, Cabianca DS, Baralle FE, Ghigna $\mathrm{C}$ and Biamonti G: HnRNP A1 controls a splicing regulatory circuit promoting mesenchymal-to-epithelial transition. Nucleic Acids Res 41: 8665-8679, 2013. 
22. Ghigna C, Giordano S, Shen H, Benvenuto F, Castiglioni F, Comoglio PM, Green MR, Riva S and Biamonti G: Cell motility is controlled by SF2/ASF through alternative splicing of the Ron protooncogene. Mol Cell 20: 881-890, 2005.

23. Lu H, Han N, Xu W, Zhu Y, Liu L, Liu S and Yang W: Screening and bioinformatics analysis of mRNA, long non-coding RNA and circular RNA expression profiles in mucoepidermoid carcinoma of salivary gland. Biochem Biophys Res Commun 508: 66-71, 2019.

24. Yang J, Sun G, Hu Y, Yang J, Shi Y, Liu H, Li C, Wang Y, Lv Z, Niu J, et al: Extracellular vesicle lncRNA metastasis-associated lung adenocarcinoma transcript 1 released from glioma stem cells modulates the inflammatory response of microglia after lipopolysaccharide stimulation through regulating miR-129-5p/high mobility group box-1 protein axis. Front Immunol 10: 3161, 2020.

25. Kun-Peng Z, Chun-Lin Z and Xiao-Long M: Antisense lncRNA FOXF1-AS1 promotes migration and invasion of osteosarcoma cells through the FOXF1/MMP-2/-9 pathway. Int J Biol Sci 13 1180-1191, 2017.

26. Zhao SJ, Kong FQ, Jie J, Li Q, Liu H, Xu AD, Yang YQ, Jiang B, Wang DD, Zhou ZQ, et al: Macrophage MSR1 promotes BMSC osteogenic differentiation and M2-like polarization by activating $\mathrm{PI} 3 \mathrm{~K} / \mathrm{AKT} / \mathrm{GSK} 3 \beta / \beta$-catenin pathway. Theranostics 10: 17-35, 2020.
27. Wu CY, Zheng C, Xia EJ, Quan RD, Hu J,Zhang XH and Hao RT: Lysophosphatidic acidreceptor5 (LPAR5) plays a significance role in papillary thyroid cancer via phosphatidylinositol 3-kinase/Akt/ mammalian target of rapamycin (mTOR) pathway. Med Sci Monit 26: e919820, 2020.

28. Jiang L, Liu XQ, Ma Q, Yang Q, Gao L, Li HD, Wang JN, Wei B, Wen J, Li J, et al: hsa-miR-500a-3P alleviates kidney injury by targeting MLKL-mediated necroptosis in renal epithelial cells. FASEB J 33: 3523-3535, 2019.

29. Xu R, Wu J, Zhang X, Zou X, Li C, Wang H, Yuan M, Chen M, Sun Q and Liu S: Modified Bu-zhong-yi-qi decoction synergies with 5 fluorouracile to inhibits gastric cancer progress via PD-1/PD-L1-dependent T cell immunization. Pharmacol Res 152: 104623, 2020.

30. Ling Y, Kuang Y, Chen LL, Lao WF, Zhu YR, Wang LQ and Wang D: A novel RON splice variant lacking exon 2 activates the PI3K/AKT pathway via PTEN phosphorylation in colorectal carcinoma cells. Oncotarget 8: 39101-39116, 2017.

This work is licensed under a Creative Commons Attribution-NonCommercial-NoDerivatives 4.0 International (CC BY-NC-ND 4.0) License. 